\title{
Giant congenital melanocytic nevus with neurocutaneous melanosis
}

\section{Lauren Petit ${ }^{1}$, Tyson Meaux²}

${ }^{1}$ LSUHSC School of Medicine, 1901 Perdido Street, New Orleans, LA 70112, USA, ${ }^{2}$ LSU Dermatology, 1542 Tulane Avenue, New Orleans, LA 70112, Suite 639, USA

Corresponding author: Lauren Petit, E-mail: 1peti1@1suhsc.edu

\begin{abstract}
Giant congenital melanocytic nevi (CNM), present at birth and greater than $40 \mathrm{~cm}$ in size, exhibit an increased risk of malignant transformation and may be associated with neurocutaneous melanosis. We present the case of a twelve month old female who presented with a giant congenital melanocytic nevus involving the upper, middle, and a portion of the lower back with multiple satellite lesions on the face, trunk, and extremities. An MRI of the brain displayed areas of hyperintensity in the cerebral hemispheres, right thalamus, and right cerebellum, consistent with neurocutaneous melanosis, however the patient was asymptomatic. Awareness of the clinical features of giant CMN is important for early detection of neurocutaneous melanosis and appropriate treatment.
\end{abstract}

Key words: Congenital; Melanocytic; Neurocutaneous

\section{INTRODUCTION}

Congenital melanocytic nevi (CMN) are typically present at birth, harbor mutations in NRAS, and can vary greatly in size. Small CMN are less than $1.5 \mathrm{~cm}$, medium-sized $\mathrm{CMN}$ are 1.5 to $20 \mathrm{~cm}$, large CMN are greater than $20 \mathrm{~cm}$, and giant CMN are greater than $40 \mathrm{~cm}$ [1]. Large CNM, which have a greater association with NRAS, have a 2-7\% increased risk of malignant transformation, often occurring before the patient reaches puberty [1]. Neurocutaneous melanosis (NCM) is a rare neuroectodermal syndrome, defined as a single giant congenital nevus or multiple congenital nevi, accompanied by meningeal melanosis or CNS melanoma with the absence of cutaneous melanoma or meningeal melanoma [2].

\section{CASE REPORT}

A 12 month old Vietnamese female with no significant past medical history presented for evaluation of a congenital melanocytic nevus that had been present since birth. Physical exam showed a darkly pigmented, hypertrichotic plaque involving the upper, middle, and a portion of the lower back, with a rim of erythema along the inferior border of the plaque and several large satellite lesions near the inferior aspect of the plaque (Fig. 1). A large, compressible pedunculated nodular growth was noted on the posterior neck within the plaque as well as numerous pigmented satellite lesions of varying size scattered on the trunk, upper and lower extremities, and face (Figs. 1 and 2). All laboratory investigations were within normal limits. An MRI of the brain showed multifocal, patchy areas of cortical hyperintensity of the cerebral hemispheres, right thalamus, and right cerebellum, consistent with NCM. The patient's family denied that the patient ever had seizures or other neurologic manifestations. There is no family history of a similar condition. Spinal MRI revealed a central enhancing nodule within the large nodule on the posterior neck, and biopsy was considered. After review by radiology at Texas Children's Hospital, the decision was made to defer biopsy and follow with MRI. The patient was then seen by pediatric hematology-oncology, who also recommended repeat MRI along with regular neurology and dermatology follow up.

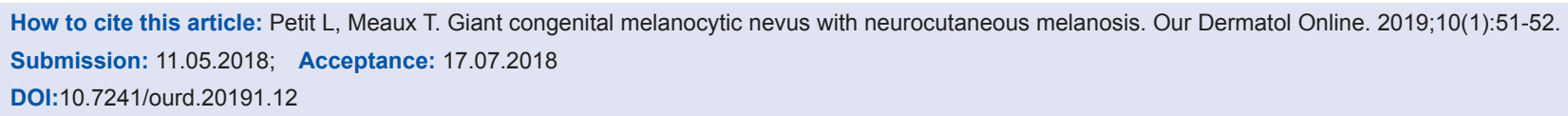




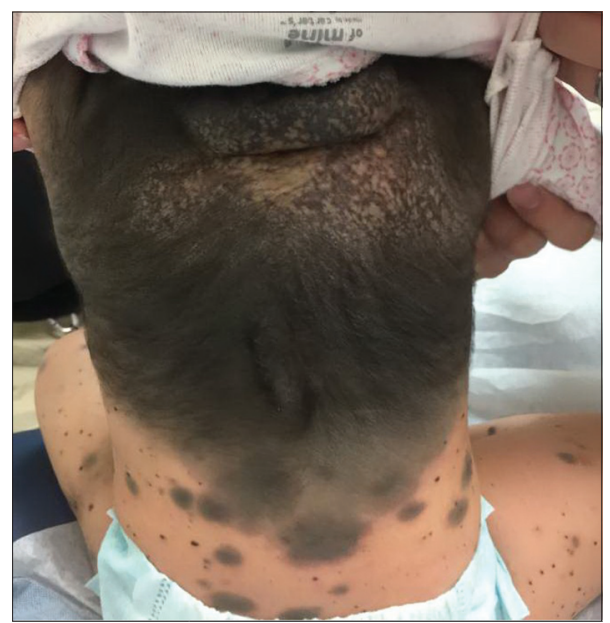

Figure 1: Giant congenital melanocytic nevus involving the upper, middle, and a portion of the lower back with multiple satellite lesions.

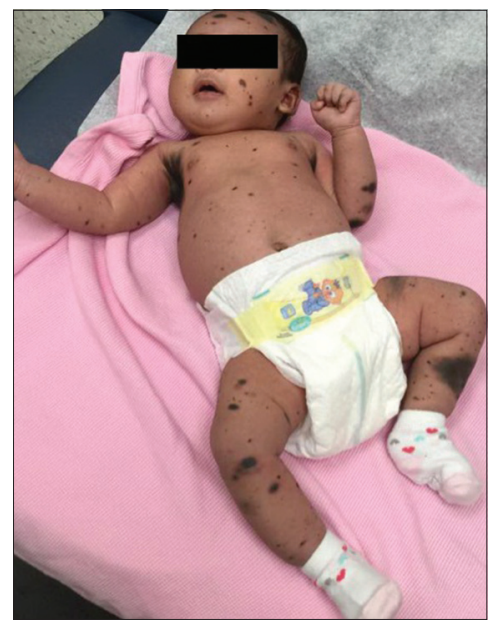

Figure 2: Multiple satellite lesions on the face, trunk, and extremities.

\section{DISCUSSION}

Large/giant $\mathrm{CMN}$ are rare, with an estimated incidence of $0.005 \%$. Symptomatic NCM may affect $6 \%$ to $11 \%$ of patients with large CMN [3]. NCM may be symptomatic or asymptomatic. While seizures are the most common initial manifestation of NCM, other possibilities include hydrocephalus, developmental delays, psychiatric disorders, cranial nerve palsies, and intracranial hemorrhage $[2,3]$. The prognosis is poor, with treatment being palliative, including ventriculoperitoneal shunts and anticonvulsants. Some reports have shown death within 2 to 3 years of symptom onset [3]. In addition to the size of CMN, anatomic location and presence of satellite nevi may be associated with a higher risk of development of NCM. In a series of 33 patients with NCM, all had a posterior axial location, and 31 had satellite nevi [4].
Therefore, MRI of the CNS should be considered in all patients with large/giant CMN with multiple satellite nevi, in addition to frequent monitoring for signs of CNS involvement [5].

The risk of melanoma in patients with $\mathrm{CMN}$ is likely related to the size, with larger nevi having a higher risk of melanoma. Large CMN have a $2.5 \%$ risk of development of melanoma, and giant CMN have a risk of $3.1 \%$. When melanoma does occur in smaller CMN, it typically begins in the epidermis, whereas melanoma arising within larger nevi may involve the dermis or subcutaneous tissue and may be more difficult to detect early [5].

Lifelong examination every 6-12 months, including palpation, of giant $\mathrm{CMN}$ is required if not completely excised, along with baseline photography. Biopsy is indicated for enlarging firm papulonodules and new areas of induration or ulceration. Surgical options include dermabrasion, curettage, Lasers (Q-switched ruby, Q-switched alexandrite), and staged excision. If surgical excision is performed, it is recommend to wait until at least six months of age to decrease the risk of adverse effects from general anesthesia [5].

\section{CONSENT}

The examination of the patient was conducted according to the Declaration of Helsinki principles.

\section{REFERENCES}

1. Barnhill RL, Cerroni L, Cook M. State of the Art, Nomenclature, and Points of Consensus and Controversy Concerning Benign Melanocytic Lesions: Outcome of an International Workshop. Adv Anat Pathol. 2010;17:73-90.

2. Kadonaga JN, Frieden IJ. Neurocutaneous melanosis: Definition and review of the literature. J Am Acad Dermatol. 1991;24:747-55.

3. Agero ALC, Benvenuto-Andrade C, Dusza SW. Asymptomatic neurocutaneous melanocytosis in patients with large congenital melanocytic nevi: A study of cases from an Internet-based registry. J Am Acad Dermatol. 2005;53:959-65.

4. Lovett A, Maari C, Decarie J-C. Large congenital melanocytic nevi and neurocutaneous melanocytosis: One pediatric center's experience. J Am Acad Dermatol. 2008;61:766-74.

5. Balin S, Barnhill R. Benign Melanocytic Neoplasms In: Bolognia JL, Schaffer JV, Cerroni L. Dermatology. $4^{\text {rd }}$ ed. Philadelphia, PA: Elsevier Saunders; 2018:chap 112:1976.

Copyright by Lauren Petit, et al. This is an open-access article distributed under the terms of the Creative Commons Attribution License, which permits unrestricted use, distribution, and reproduction in any medium, provided the original author and source are credited.

Source of Support: Nil, Conflict of Interest: None declared. 